\title{
The Establishment of Tetraploid Human Embryonic Cells Using Demecolcine
}

\author{
Minoru Miyagoshi*, Kohzaburo Fujikawa-Yamamoto, and Hiroko Yamagishi \\ Division of Cell Medicine, Research Institute of Medical Science, \\ Kanazawa Medical University, Ishikawa 920-0293, Japan \\ Received September 5, 2013; accepted October 10, 2013
}

\begin{abstract}
Summary Diploid human embryonic cells were exposed to demecolcine for $3 \mathrm{~d}$ and then released from this exposure. At that time, the cell population was a mixture of diploid and tetraploid cells. Tetraploid cells were obtained through the cloning of this mixture of cells. The doubling time of the tetraploid cells was $16 \mathrm{~h}$, identical to that of the diploid cells. The tetraploid cells had 92 chromosomes and double the DNA content of the parent diploid cells. The cell volume of the tetraploid cells was about twice that of the diploid cells. The duration of the $\mathrm{G}_{1}, \mathrm{~S}$, and $\mathrm{G}_{2} / \mathrm{M}$ phases was almost the same as for diploid cells. It was concluded that the established tetraploid human embryonic cells have cellular characteristics similar to those of diploid human embryonic cells.
\end{abstract}

Key words Demecolcine, Human embryonic cell, Tetraploid cells, Diploid cell.

Human embryonic cells have been used as normal cells transfected with the hTERT (Human Telomerase Reverse Transcriptase) gene. They can be polyploidized by demecolcine, and some of the cells become tetraploid.

Demecolcine antagonizes tubulin polymerization and induces disassembly of microtubules into monomers (Inoue 1981). The drug inhibits spindle fiber formation in the M phase and polyploidizes many cells. We have reported that V79 and B16-F10 cells were polyploidized by demecolcine at a wide range of concentrations, but that HeLa-S3, L1210, K562, and B16 melanoma cells were not (Fujikawa-Yamamoto et al. 1993). Tetraploid and octaploid Meth-A cell lines have been established from diploid and tetraploid cells using demecolcine (Fujikawa-Yamamoto et al. 2003). They exhibit different characteristics from each other (Fujikawa-Yamamoto et al. 2001, FujikawaYamamoto et al. 2002). Although polyploidization of mammalian cells occurs in various organs, particularly in an aged or partially hepatectomized liver, the mechanisms are poorly understood (Mossin et al. 1994, Zong et al. 1994, Fogt and Nanji 1996, Seglen 1997). The critical requirements for polyploidization by demecolcine have not yet been established.

In this study, a tetraploid human embryonic cell line was established from diploid cells after removal of demecolcine, and its cellular characteristics were examined.

Materials and methods

Cells

Human embryonic cells transfected with the hTERT gene were purchased from Health Science Research Resources Bank (HSRRB). The cells were maintained in a humidified atmosphere of $5 \% \mathrm{CO}_{2}$ at $37^{\circ} \mathrm{C}$ as a monolayer culture in Dulbecco's modified Eagle's medium (Wako Pure Chemical Industries, Ltd., Japan) supplemented with 10\% fetal bovine serum (Sigma, St.

*Corresponding author, e-mail: miyako@kanazawa-med.ac.jp

DOI: $10.1508 /$ cytologia. 78.417 
Louis, MO, USA), streptomycin (100 $\mu \mathrm{g} / \mathrm{ml}$, Nacalai Tesque Co., Japan), and penicillin (50 units/ $\mathrm{ml}$, Nacalai Tesque Co.).

\section{Drug treatment and cell cloning}

Exponentially growing cells were plated into culture flasks $\left(25 \mathrm{~cm}^{2}\right.$; Corning Costar Acton, MA, USA) at a density of about $1 \times 10^{6}$ cells/flask, and the medium was changed $24 \mathrm{~h}$ after seeding. Twelve hours thereafter, the cells were exposed to demecolcine $(270 \mathrm{nM}$, Sigma) for $3 \mathrm{~d}$. The cells were then released from the drug exposure by changing the medium. Every 2 days, the cells were subcultured at a $1 / 2$ dilution by two washes with divalent cation-free phosphate-buffered saline $\left(\mathrm{PBS}^{-}\right)$and subsequent trypsinization $(0.2 \%$ trypsin and $30 \mathrm{mM}$ EDTA). The residual cells were prepared for flow cytometry (FCM).

At 30 days after exposure to demecolcine, the cell population was a mixture of diploid and tetraploid cells, and the cells were seeded in 96-well dishes (96-well tissue cultures, $6.4 \mathrm{~mm}$ diameter, Corning Costar Co., Cambridge, MA, USA) at low density. The wells containing a cell were selected. The cells were incubated in a $\mathrm{CO}_{2}$ incubator with occasional changes of medium. About 10 days later, the cells were trypsinized, transferred into culture flasks, and cultured again. Cellular DNA content was checked by FCM when the cells had grown sufficiently to obtain DNA histograms. The first measurement for DNA histograms was performed about two months after the removal of demecolcine, and the expected number of cell divisions was above 20 at that time. Tetraploid human embryonic cells were cloned.

\section{Cell preparation for flow cytometry (FCM) and cell counting}

The cells were fixed with $20 \%$ ethanol, and then incubated with $0.5 \mathrm{ml}$ of $0.25 \%$ RNase (Type II-A, Sigma) for $3 \mathrm{~h}$ at $4^{\circ} \mathrm{C}$. The cell number was counted with a hemocytometer at this stage. Then, the cells were stained with propidium iodide (PI, $\left.7.5 \times 10^{-5} \mathrm{M}\right)$ and examined for red fluorescence by FCM. Under these staining conditions, the signal due to residual double-stranded RNA was negligible and the relative intensity of the red fluorescence corresponded to the DNA content (Krishan 1975).

\section{FCM measurements}

The fluorescence from individual cells was measured using a FACSCalibur (Becton-Dickinson Immunocytometry Systems, Franklin Lakes, ND, USA). The fluorescence of individual cells irradiated with focused laser light at a wavelength of $488 \mathrm{~nm}$ was detected using a photomultiplier tube. The relative intensity of red fluorescence (FL2A and FL2H) was measured and DNA histograms were obtained.

\section{Cell cycle analysis}

Flow cytometry (FCM) data of FL2A (signals of red-fluorescence intensity processed through a linear amplifier) for 10,000 cells were entered into CASL software (Mathematica, Wolfram Research Inc., Champaign, IL, USA) for cell cycle analysis of DNA histograms on a log scale using the transfer software "FACS to ASCII" (freeware), and the DNA histograms were decomposed to phase fractions based on DNA content (Fujikawa-Yamamoto and Miyagoshi 2006). The algorithm of LISCA is similar to Fried's method (Fried 1977).

\section{Chromosome analysis}

Exponentially growing cells were exposed to demecolcine at a concentration of $270 \mathrm{nM}$ for $1 \mathrm{~h}$. The cells were trypsinized, swelled with $75 \mathrm{mM} \mathrm{KCl}$, fixed with fixing solution $\left(\mathrm{CH}_{3} \mathrm{OH}: \mathrm{CH}_{3} \mathrm{COOH}=7: 3\right)$, and dropped onto glass slides. The cells were stained with Giemsa solution prior to photographing the chromosomes. Chromosome numbers were counted from the 
photographs.

\section{Cell volume distribution}

The exponentially growing cells were trypsinized, fixed with $20 \%$ ethanol, and resuspended in $\operatorname{PBS}^{(-)}$. The distribution of cell volume (Coulter volume) was measured using a Coulter Counter (ZM/256, Coulter Electronics, Fullerton, CA, USA). Standard spheres $(9.8 \mu \mathrm{m}$ diameter, Coulter sphere, Coulter Electronics) were used as control. Note that the Coulter volume depends on the material tested because it is calculated based on the resistance of particles.

\section{Cell morphology}

The morphology of exponentially growing cells in Lab-Tek Chamber Slides (Nalge Nunc International, Cambridge, MA, USA) was photographed using a phase-contrast microscope (Nikon, Tokyo, Japan) equipped with a digital camera system (Moticam 2000, Simadzu-rika Co., Japan).
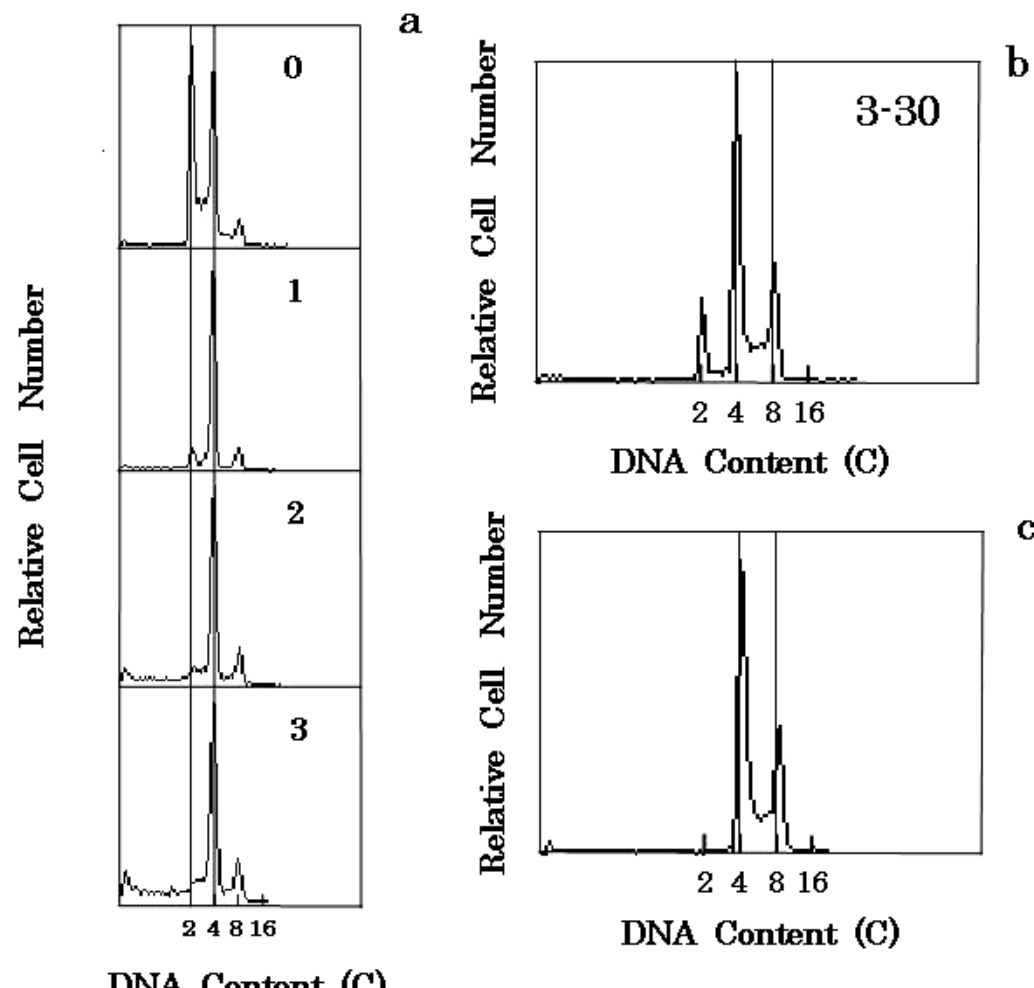

DNA Content (C)

DNA Content (C)

Fig. 1. Changes in DNA fluorescence histograms of diploid human embryonic cells after the addition and removal of demecolcine. Exponentially growing diploid human embryonic cells were exposed to demecolcine at a concentration of $270 \mathrm{nM}$ for 1,2 , and 3 days (a). The cells were released from the drug and cultured again with occasional subculturing. Paired numerals in the histogram represent the time (days) of the drug removal and time after removal, in that order (b). The population of diploid and tetraploid cells was one-cell cloned, and tetraploid clones were obtained (c). They were gathered into one cell line and used as a tetraploid human embryonic cell population. The abscissa represents the relative DNA content (C, complement). Vertical black lines are included to facilitate understanding. 


\section{Results}

To examine the polyploidization caused by demecolcine, diploid human embryonic cells were exposed to it for 3 days. The DNA content of the main population of diploid human embryonic cells was $4 \mathrm{c}$ at 3 days after addition of the drug. At 30 days after drug removal, the population was a mixture of cells with peaks of 2, 4, and $8 \mathrm{c}$ DNA content, suggesting a diploid/tetraploid cell mixture. At 30 days, the cells were seeded into multi-well plates to obtain monoclonal tetraploid lines. After a month, a monoclonal cell population was obtained (Fig. 1).

To examine the growth of tetraploid human embryonic cells, the cell numbers were counted, corrected for the subcultures, and plotted against days. Figure 2 shows the growth curves of longterm culturing (left panel). The doubling times of the cell populations were $16.0 \mathrm{~h}$ for both diploid and tetraploid human embryonic cells. We thus concluded that the doubling times were almost the same for diploid and tetraploid human embryonic cells under these experimental conditions.

Representative outputs of CASL for DNA histograms of exponentially growing diploid and tetraploid human embryonic cells are shown in Fig. 2 (right panel). The DNA content measured from the fluorescence intensity of PI intercalated in double-stranded DNA was about twice that of diploid cells. The phase fractions obtained through CASL are listed in Table 1. No remarkable difference was observed in the cell cycle parameters of diploid and tetraploid human embryonic cells, although a $0.4 \mathrm{~h}$ elongation of the $\mathrm{S}$ phase in tetraploid cells was observed. The cell cycle parameters of diploid and tetraploid human embryonic cells were the same, at least under our experimental conditions, suggesting that the rate of DNA synthesis was in proportion to DNA content.

To examine the morphological characteristics of the diploid and tetraploid human embryonic cells, phase-contrast micrographs and the cell volume (Coulter volume) were compared with those of diploid cells (Fig. 3). The ratio of cell volume for diploid and tetraploid human embryonic cells
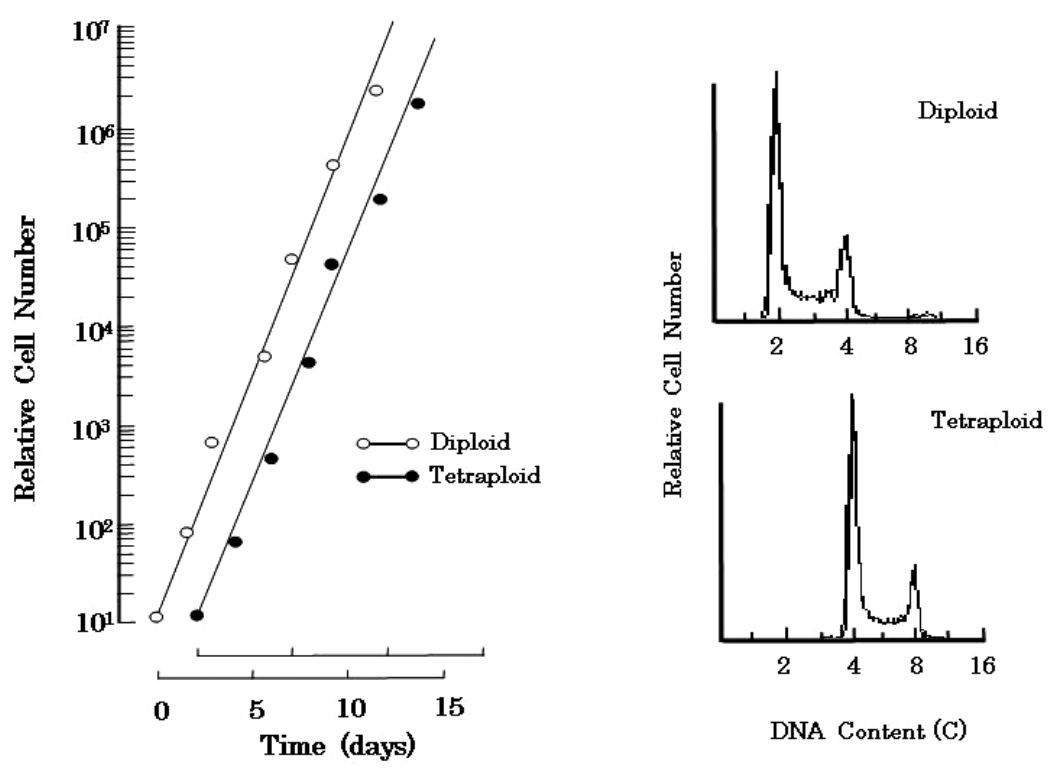

Fig. 2. Growth curves (left panel) and representative DNA fluorescence histograms (right panel) of diploid and tetraploid human embryonic cells after cloning. Exponentially growing diploid and tetraploid human embryonic cells were cultured for the long term with 2-day subcultures. Changes in the relative cell numbers of diploid (open circle) and tetraploid (closed circle) human embryonic cells were determined. The abscissa represents the time (day) after cloning. Solid lines are included to facilitate understanding. In the right panel, solid (black) and dotted (gray) lines represent experimental and synthesized histograms, respectively. 
Table 1. Cell cycle parameters of diploid and tetraploid human embryonic cells

\begin{tabular}{llll}
\hline \hline \multicolumn{1}{c}{ Phase } & $\mathrm{G}_{1}$ & $\mathrm{~S}$ & $\mathrm{G}_{2} / \mathrm{M}$ \\
\hline Diploid cells (Td=16.0h) & & & \\
$\quad$ Fractiion & 0.49 & 0.31 & 0.20 \\
$\quad$ Duration (h) & 6.5 & 5.3 & 4.2 \\
Tetraploid cells (Td=16.0h) & & & \\
$\quad$ Fraction & 0.56 & 0.28 & 0.17 \\
$\quad$ Duration (h) & 7.5 & 4.9 & 3.6 \\
\hline
\end{tabular}

* Phase fractions of diploid and tetraploid cells were determined omitting those for other ploidies in the human embryonic cell population.

** Phase duration was calculated through conventional equations using doubling time instead of cycle time (Watanabe and Okada 1967). Td, doubling time calculated from Fig. 2.
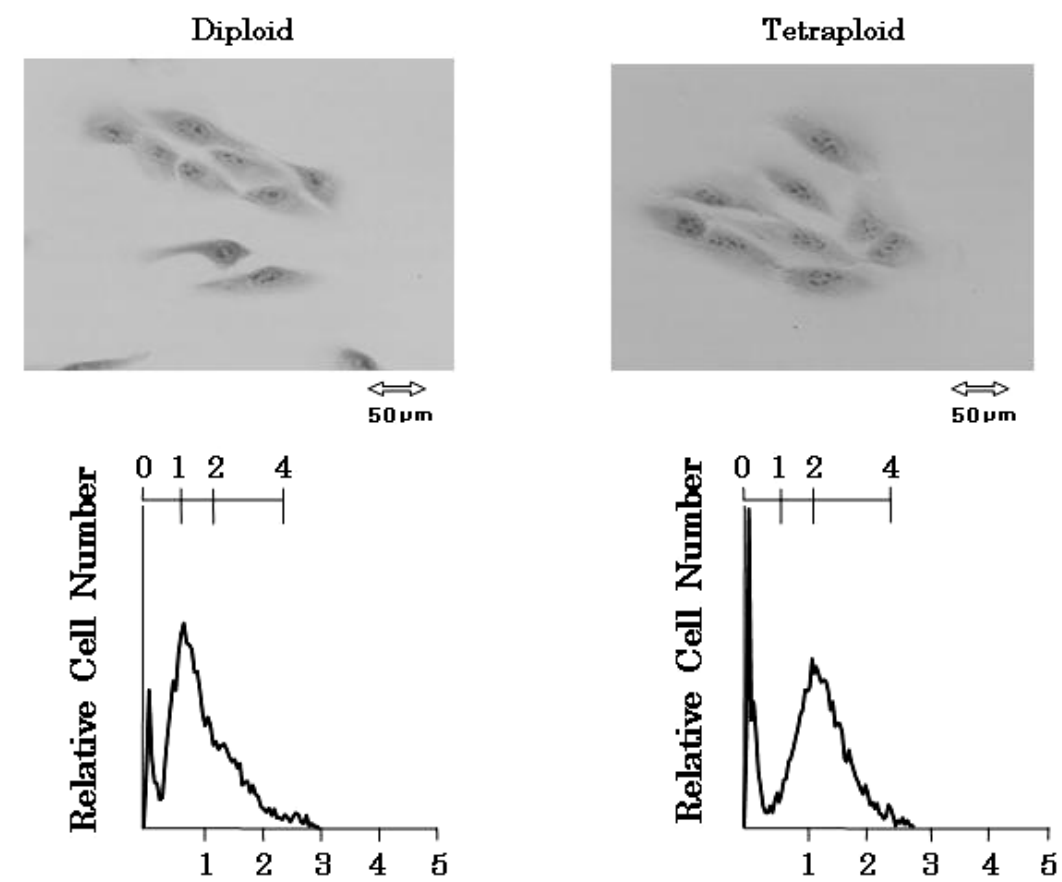

\section{Relative Channel Number}

Fig. 3. Micrographs (upper panels) and volume distribution (lower panels) of diploid (left panel) and tetraploid (right panel) human embryonic cells. Exponentially growing diploid and tetraploid human embryonic cells were stained with hematoxylin and eosin.

was about $1: 2$, suggesting that pluripotent potential was preserved during the diploid-tetraploid transition.

To examine the integrity of diploid and tetraploid human embryonic cells, chromosome number was counted and the distribution was obtained (Fig. 4). The chromosome numbers of diploid and tetraploid cells were 46 and 92, respectively, proving that these cells were indeed diploid and tetraploid. This means that the chromosome number was doubled in the polyploidization process.

\section{Discussion}

Several polyploid cell lines have been established from highly polyploidized cells (Fujikawa- 
Diploid
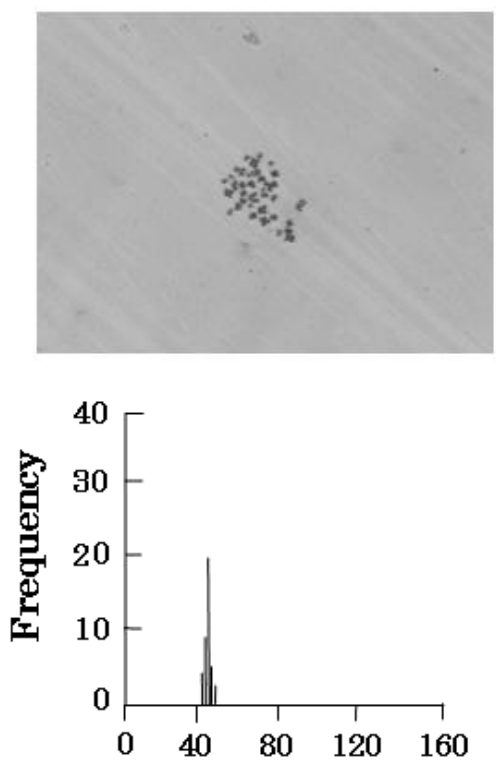

Tetraploid
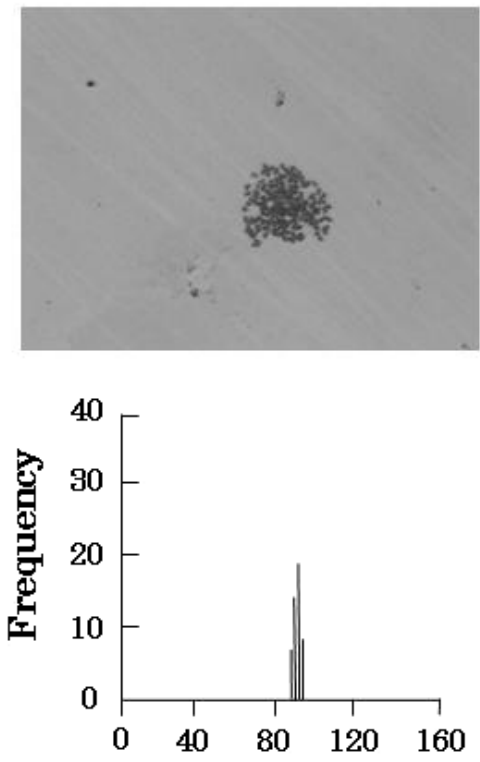

\section{Chromosome Number}

Fig. 4. Micrographs (upper panels) and histograms of chromosome number (lower panels) of diploid (left panel) and tetraploid (right panel) human embryonic cells. Exponentially growing diploid and tetraploid human embryonic cells were exposed to demecolcine at a concentration of $270 \mathrm{nM}$ for $1 \mathrm{~h}$. The chromosomes were stained with Giemsa solution. The chromosomes of about 50 cells were enumerated from enlarged photographs.

Yamamoto et al. 2003, Fujikawa-Yamamoto et al. 2007, Miyagosh et al. 2007). However, because there is finite proliferation ability in normal human fibroblasts, aging disturbs the observed progress in long-term culture and isolation cultivation by cloning, so the characteristics of established polyploid cells of human fibroblasts remain unknown. The mechanism of ploidy transition is not yet known, but it has been proposed that the genome structures of polyploidized and polyploid cells are distinctly different (Fujikawa-Yamamoto 2006). In this study, the main population of diploid human embryo cells became a mixture of diploid and tetraploid cells after demecolcine removal. Tetraploid cells were obtained through the cloning of this mixture of cells. Tetraploid cells might be necessary to rebuild the chromosome composition for the establishment of polyploid cells.

The doubling time of the population was $16 \mathrm{~h}$ for both diploid and tetraploid cells. It is of interest that the doubling time was almost the same in diploid and tetraploid human embryo cells. Although two rates of DNA synthesis, constant and double-increase, have been reported for polyploid cells, the doubling time increased with the ploidy in both cases (Fujikawa-Yamamoto et al. 2000). If the intracellular content increases with the ploidy of human embryo cells, a spatial increase would cause a delay in the transport of materials in highly polyploid cells. However, cell cycle parameters were almost the same between diploid and tetraploid cells (Table 1), suggesting that the rate of DNA synthesis is about two-fold faster in tetraploid cells than in diploid cells. Although tetraploid cells are not major cell constituents in mammals, the results obtained here suggest than increases in ploidy and cell volume do not impair cell survival. It seems that there is high stability of ploidy in tetraploid cells. 


\section{References}

Fogt, F., and Nanji, A. A. 1996. Alterations in nuclear ploidy and cell phase distribution of rat liver cells in experimental alcoholic liver disease: relationship to antioxidant enzyme gene expression. Toxicol. Appl. Pharmacol. 136: 87-93.

Fried, J. 1977. Analysis of deoxyribonucleic acid histograms from flowcyto fluorometry. Estimation of distribution of cells within S phase. J. Histochem. Cytochem. 25: 942-951.

Fujikawa-Yamamoto, K. 2006. Anhypothesis about genome structures in mammalian polyploidy cells based on a new concept that genome is fractal of six hierarchies. Med. Hypotheses 66: 337-344.

Fujikawa-Yamamoto, K., Ikeda, T., Wang, S., Yamagishi, H., and Miyagoshi, M. 2002. Serum dependence in proliferation of diploid and tetraploid Meth-A cells. Cytologia 67: 75-82.

Fujikawa-Yamamoto, K., Iwai, H., Teraoka, K., Odashima, S., and Murakami, E. 1993. Typing of responses of cultured cells to demecolcine and critical control point in the M phase. J. Kanazawa Med. Univ. 18: 98-105.

Fujikawa-Yamamoto, K., and Miyagoshi, M. 2006. Cell cycle analysis using flow cytometry III-Stathomokinetic analysis-. Cytometry Res. 16: 43-49.

Fujikawa-Yamamoto, K., Miyagoshi, M., and Yamagishi, H. 2007. Establishment of tetraploid cell line from mouse H-1(ES) cells highly polyploidized with demecolcine. Cell Prolif. 40: 327-337.

Fujikawa-Yamamoto, K., Wang, S. Yamagishi, H., and Miyagoshi, M. 2001. Temperature dependence in proliferation of tetraploid Meth-A cells in comparison with the parent diploid cells. Cell Struct. Funct. 26: 263-269.

Fujikawa-Yamamoto, K., Yamagishi, H., and Miyagoshi, M. 2003. Octaploid Meth-A cells are establishrd from highly polyploidized cell population. Cell Prolif. 36: 87-100.

Fujikawa-Yamamoto, K., Yamagishi, H., Zong, Z., Ohdoi, C., and Wang, S. 2000. Different responses of polyploidized V79 cells after removal of two drugs, demecolcine and K252a. Cell Struct. Funct. 25: 41-46.

Inoue, S. 1981. Cell division and mitotic spindle. J. Cell Biol. 91: 131-147.

Krishan, A. 1975. Rapid flow cytofluorometric analysis of mammalian cell cycle by propidium iodide staining. J. Cell Biol. 66: 188-193.

Miyagoshi, M., Fujikawa-Yamamoto, K., and Yamagishi, H. 2007. The establishment of tetraploid V79 cells using demecolcine. J. Kanazawa Med. Univ. 32: 170-175.

Mossin, L., Blankson, H., Huitfeldt, H., and Seglen, P. O. 1994. Ploidy-dependent growth and binucleation in cultured rat hepatocytes. Exp. Cell Res. 214: 551-560.

Seglen, P. O. 1997. DNA ploidy and autophagic protein degradation as determinant of hepatocellular growth and survival. Cell Biol. Toxicol. 13: 301-315.

Watanabe, I., and Okada, S. 1967. Effects of temperature on growth rate of cultured mammalian cells (L1578Y). J. Cell Biol. 32: 309-323.

Zong, Z., Fujikawa-Yamamoto, K., Teraoka, K., Yamagishi, H., Tanino, M., and Odashima, S. 1994. Potentiation of K-252a, a protein kinase inhibitor-induced polyploidization by cAMP in cultured fibrosarcoma cell line. Biochem. Biophys. Res. Commun. 205: 745-750. 